\title{
Reabsorción de nitrógeno en gramíneas perennes de diferente palatabilidad, nativas del Caldenal
}

\author{
Alicia S. Moretto ${ }^{1, \bowtie}$; Nilda G. Didoné2 \& Roberto A. Distel ${ }^{2,3}$ \\ 1. Centro Austral de Investigaciones Científicas (CONICET). Ushuaia, Argentina. 2. Centro de Recursos Naturales Renovables \\ de la Zona Semiárida (CONICET). Bahía Blanca, Argentina. 3. Departamento de Agronomía (Universidad Nacional del Sur). \\ Bahía Blanca, Argentina.
}

\begin{abstract}
Resumen. La reabsorción de nutrientes en las plantas permite su conservación y uso en la construcción de nuevos tejidos, por lo cual podría esperarse que fuera más acentuada en especies adaptadas a crecer con baja disponibilidad de nutrientes. En el Distrito del Caldén, las gramíneas de palatabilidad baja reúnen características distintivas de especies adaptadas a condiciones de poca fertilidad (e.g., tejido esclerenquimatoso abundante, baja concentración de nutrientes), características que en las gramíneas palatables son menos manifiestas. Por lo tanto, cabría esperar una mayor eficiencia y/o proficiencia de reabsorción y de utilización de nitrógeno (N) en Nassella tenuissima Trin. (palatabilidad baja) que en Poa ligularis Nees ex. Steud. (palatabilidad alta). El objetivo de este trabajo fue comparar la dinámica del $\mathrm{N}$ en hojas y raíces, vivas y muertas, de ambas especies en distintos momentos del ciclo anual de crecimiento. La concentración de $\mathrm{N}$ en los compartimientos analizados fue similar entre especies, o mayor en $P$. ligularis. Esto determinó una eficiencia y/o proficiencia de reabsorción y de utilización de $\mathrm{N}$ similar entre especies, o mayor en $N$. tenuissima que en P. ligularis. En ambas especies, la eficiencia de reabsorción de $\mathrm{N}$ promedio fue mayor a nivel foliar que a nivel radical. Los resultados obtenidos no permiten inferir un comportamiento claramente diferencial en la economía del uso de $\mathrm{N}$ entre las gramíneas de baja y de alta palatabilidad del Caldenal.
\end{abstract}

[Palabras clave: eficiencia de reabsorción de nitrógeno, proficiencia de reabsorción de nitrógeno, eficiencia de utilización de nitrógeno, Poa ligularis, Nassella tenuissima]

\begin{abstract}
Aвstract. Nitrogen resorption in perennial grasses with different palatability, native to the Caldenal: Nutrient resorption in plants represents a conservation mechanism which could be expected to be more significant in those species adapted to nutrient limitations. In the Caldenal District, unpalatable grasses meet characteristics commonly present in species adapted to unfertile conditions (abundance of structural tissue, low nutrient concentration), whereas in palatable grasses those traits are less manifest. So, it could be expected a higher nitrogen $(\mathrm{N})$ resorption efficiency and/or proficiency and $\mathrm{N}$ use efficiency in Nassella tenuissima Trin. (unpalatable grass) than in Poa ligularis Nees ex. Steud. (palatable grass). Our objective was to compare the N concentration in leaves and roots, live and death, of both species at different times along an annual growth cycle. Nitrogen concentration in all plant compartments was similar between both species or higher in P. ligularis, determining similar or higher $\mathrm{N}$ resorption efficiency and proficiency and $\mathrm{N}$ use efficiency in $\mathrm{N}$. tenuissima than in P. ligularis. Average $\mathrm{N}$ resorption efficiency was higher in leaves that in roots for both species. Our results did not allow inferring a clear differential behavior in $\mathrm{N}$ use economy between these unpalatable and palatable grasses of the Caldenal.
\end{abstract}

[Keywords: nitrogen resorption efficiency, nitrogen resorption proficiency, nitrogen use efficiency, Poa ligularis, Nassella tenuissima]

\section{INTRODUCCIÓN}

La reabsorción de nutrientes en las plantas consiste en su removilización desde los tejidos envejecidos hacia los órganos de reserva o hacia los tejidos en crecimiento activo. Este proceso puede cuantificarse a través de la eficiencia de reabsorción (proporción del nutriente reabsorbido del tejido senescente) o mediante la proficiencia de reabsorción (concentración mínima del nutriente en el tejido senescente) (Killingbeck 1996). Las plantas perennes pueden reabsorber, en promedio, $50 \%(41.4-58.5 \%)$ del nitrógeno y $52 \%(42.4-71.5 \%)$ del fósforo antes de la abscisión de las hojas (Aerts 1996), lo que

Centro Austral de Investigaciones Científicas (CONICET). Ushuaia, Argentina. cadicmoretto@gmx.net; amoretto@cadic-conicet.gob.ar permite su conservación y posterior utilización para la construcción de nuevos tejidos.

La reabsorción constituye un mecanismo de conservación de nutrientes, por lo que podría esperarse que las plantas adaptadas a condiciones de baja disponibilidad de nutrientes presenten una mayor eficiencia y/o proficiencia de reabsorción (Grime 1979; Chapin 1980). Sin embargo, algunos estudios han mostrado que la eficiencia de reabsorción no siempre es mayor en las especies adaptadas a condiciones de baja fertilidad, aunque sí han puesto de manifiesto una mayor proficiencia de reabsorción (Aerts 1996; Killingbeck 1996; Aerts \& Chapin 2000; Wright \& Westoby 2003).

Recibido: 24 de abril de 2012; Fin de arbitraje: 23 de julio; Revisión recibida: 22 de agosto; Aceptado: 3 de septiembre. 
Dado que una parte importante del total de los nutrientes minerales se encuentra en las hojas, y que su estudio resulta más sencillo en comparación con las raíces, no resulta extraño que el proceso de reabsorción de nutrientes se haya estudiado con mayor intensidad en los tejidos foliares que en los subterráneos (Aerts 1996; Aerts \& Chapin 2000). Aunque por lo general la eficiencia de reabsorción de nutrientes en las raíces se supone relativamente baja (Oestertag \& Hobbie 1999; Wedin 1999), las mediciones cobran particular importancia en ecosistemas de regiones áridas y semiáridas, donde una proporción elevada de las biomasa total está representada por la biomasa radical (Caldwell 1979).

En el Distrito del Caldén (Cabrera 1976; de aquí en adelante referido como "Caldenal") las gramíneas de palatabilidad baja presentan menores concentraciones de nutrientes y mayores de fibra y lignina, en comparación con las gramíneas de palatabilidad alta (Cano 1988; Cerqueira et al. 2004; Distel et al. 2005). Estas características permiten inferir una mayor adaptación para crecer en condiciones de baja disponibilidad de nutrientes en las gramíneas de baja palatabilidad que en las de alta palatabilidad (Grime 2001). En consecuencia, se puede esperar que las gramíneas de baja palatabilidad presenten una mayor economía en el uso de nutrientes que las de alta palatabilidad. La hipótesis de trabajo del presente estudio establece que la eficiencia y/o proficiencia de reabsorción de nitrógeno (N) es mayor en Nassella tenuissima Trin. (baja palatabilidad) que en Poa ligularis Nees ex. Steud. (alta palatabilidad). Nos concentramos en estudiar la reabsorción de $\mathrm{N}$ dado que este nutriente representa uno de los principales factores limitantes del crecimiento de las plantas en los ecosistemas naturales (Chapin 1980). Determinamos la concentración de N en hojas y raíces, vivas y muertas, de $P$. ligularis y $N$. tenuissima en distintos momentos del ciclo anual de crecimiento, con el fin de cuantificar la proficiencia y la eficiencia de reabsorción de $\mathrm{N}$ en ambas especies. Además, calculamos la eficiencia de uso del $\mathrm{N}$, esperando encontrar mayores valores de eficiencia en la gramínea de baja palatabilidad. La eficiencia de uso de $\mathrm{N}$ se definió originalmente como la tasa de flujo entre producción de materia seca y absorción de N (Vitousek 1982), y más tarde como el producto entre la productividad del N (tasa de producción de materia seca por unidad de N) y el tiempo medio de residencia del $\mathrm{N}$ en la planta (lapso durante el cual el $\mathrm{N}$ está disponible para la fijación de carbono) (Berense \& Aerts 1987).

\section{MATERIALES y MÉTODOS}

Las muestras de hojas y raíces de P. ligularis y $N$. tenuissima fueron recolactadas en un sitio excluido del pastoreo del ganado doméstico (20 años de clausura) localizado en el sureste de la Provincia

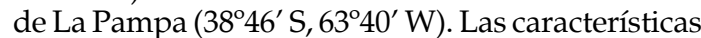
generales de la región referentes a clima, suelo y vegetación referidas a continuación han sido descriptas por Cano (1988) e INTA et al. (1980). El clima es templado, semiárido. La temperatura media anual del aire es $15^{\circ} \mathrm{C}$. El promedio histórico de las precipitaciones anuales en la región es 344 $\mathrm{mm}(\mathrm{CV}=31 \%)$. El otoño y la primavera son las estaciones más húmedas del año. El déficit de agua anual es de $\sim 400 \mathrm{~mm}$. Durante el período de estudio (2001), las precipitaciones totalizaron 744 $\mathrm{mm}$, superando los promedios mensuales históricos en los meses de otoño y primavera.

En las planicies, donde estuvo localizado el sitio de estudio, los suelos dominantes se clasifican como Calciustoles Petrocálcicos. Presentan textura franco arenosa a franco-limosa, y profundidad variable (0.60-0.80 m). El contenido de materia orgánica oscila entre 2 y $3 \%$.

La región donde se localiza el sitio de estudio pertenece al Distrito Fitogeográfico del Caldén, en su transición con la Provincia del Monte (Cabrera 1976). La fisonomía de la vegetación es de "pastizal bajo con arbustos aislados". Las especies del estrato herbáceo están representadas en mayor o menor medida (dependiendo de la historia de pastoreo) por Poa ligularis Nees ex Steud., Nassella longiglumis (Phil.) Barkworth (sinónimo Stipa clarazii), Piptochaetium napostaense (Speg.) Hack., N. tenuis (Phil.) Barkworth (sinónimo Stipa tenuis), Jarava ichu Ruiz \& Pav. (sinónimo Stipa gynerioides), N. tenuissima (Trin.) Barkworth (sinónimo Stipa tenuissima), Amelichloa brachychaeta (Godr.) Arriaga \& Barkworth (sinónimo Stipa brachychaeta), A. ambigua (Speg.) Arriaga \& Barkworth (sinónimo Stipa ambigua), Medicago minima (L.) Bartal., Erodium cicutarium (L.) L`Hér. Ex Aiton, Hordeum spp.y Bromus spp. En el estrato arbóreo/arbustivo comúnmente dominan Prosopis caldenia Burkart, P. flexuosa DC., Larrea divaricada Cav. y Condalia microphylla Cav. La nomenclatura sigue a la del Instituto de Botánica Darwinion (2009). En el estrato herbáceo del sitio de estudio, $P$. ligularis es una de las especies más abundantes, mientras que $N$. tenuissima se presenta como especie acompañante (Distel \& Boo 1996). Ambas especies cumplen el estadio vegetativo durante el período otoño-invierno-comienzo de primavera, y el reproductivo hacia fines de la primavera (Distel \& Peláez 1985). Los picos de crecimiento vegetativo ocurren en otoño e inicios de primavera, y el pico de senescencia hacia fines de primavera.

En marzo de 2001 (inmediatamente antes del inicio del ciclo anual de crecimiento) fueron seleccionadas en el sitio de estudio (clausura de 20 ha) 60 plantas de $P$. ligularis y 60 plantas de $N$. tenuissima. Las plantas fueron elegidas al azar entre las de tamaño $(10 \pm 2 \mathrm{~cm}$ de diámetro basal $)$ y forma 
modales, establecidas en espacios entre arbustos. En ese momento, todas las plantas recibieron un corte de limpieza ( $5 \mathrm{~cm}$ sobre el nivel del suelo) con el fin de eliminar material muerto en pie proveniente de ciclos de crecimiento anteriores; esto facilitó la discriminación entre material vivo y material muerto reciente. Debido a que al momento de realizar el corte de limpieza ambas especies no habían comenzado el ciclo anual de crecimiento, puede suponerse una escasa interferencia en el crecimiento aéreo y subterráneo. Entre junio y noviembre del mismo año fue cosechada la biomasa aérea y la subterránea de 10 plantas (sin cosecha previa) de cada especie, elegidas al azar al comienzo de las estimaciones. La biomasa aérea se cortó al ras del suelo, mientras que la biomasa subterránea se obtuvo mediante la extracción de un cilindro de suelo (10 cm de diámetro y $60 \mathrm{~cm}$ de profundidad) centrado en la corona de cada planta cosechada y unidas a ella.

De cada cosecha de biomasa se obtuvieron hojas y raíces, vivas y muertas. El criterio para clasificar como hoja viva fue el de lámina totalmente expandida (lígula visible) y coloración verde en toda su extensión, y como hoja muerta reciente el de lámina completamente senescente y de color amarillo en toda su extensión. Las raíces se separaron del suelo por flotación en agua de red y tamizado, proceso que se repitió hasta la extracción total de las raíces de la muestra de suelo (Distel 1987). Inmediatamente después fueron descartadas las raíces extrañas que se diferenciaban de manera clara de aquellas unidas a la corona de la planta cosechada. Del total de raíces se tomó una muestra representativa y en cantidad tal que posibilitara la determinación de $\mathrm{N}$ en la fracción viva y en la muerta. La separación de las raíces en vivas y muertas recientes se realizó por color y textura (Bohm 1979), lo cual fue comprobado en una submuestra de cada fracción mediante la prueba de reacción colorimétrica con solución de Cloruro de Trifenil Tetrazolium (Moore 1962). Para analizar raíces muertas recientes, la incertidumbre respecto al tiempo transcurrido desde la muerte fue en parte disminuida al descartar aquellas con signos de deterioro por descomposición.

El material obtenido fue secado en estufa a $60{ }^{\circ} \mathrm{C}$ hasta peso constante, y posteriormente fue molido hasta atravesar un tamiz de $1 \mathrm{~mm}$. La concentración de $\mathrm{N}$ fue determinada por el método semi-micro Kjeldahl. A partir de los valores de concentración de $\mathrm{N}$ en hojas y raíces, vivas y muertas, se calculó la eficiencia de reabsorción de N (Killingbeck 1996) y la eficiencia de uso de N (Vitousek 1982), a nivel foliar y radical, según las siguientes ecuaciones:

Eficiencia de reabsorción de $\mathrm{N}(\%)=\left[\left(\mathrm{N}_{\mathrm{v}}-\mathrm{N}_{\mathrm{m}}\right) / \mathrm{N}_{\mathrm{v}}\right] \times 100$

Eficiencia de uso de $\mathrm{N}(\mathrm{g} \mathrm{MS} / \mathrm{mg} \mathrm{N})=1 /\left[\mathrm{N}_{\mathrm{v}} \times(1-r)\right]$

donde $\mathrm{N}_{\mathrm{v}}$ es la concentración de $\mathrm{N}$ en tejido vivo, $\mathrm{N}_{\mathrm{m}}$ es la concentración de $\mathrm{N}$ en tejido muerto y $r$ es la eficiencia de reabsorción de $\mathrm{N}$ expresada como fracción. Por otra parte, la concentración de $\mathrm{N}$ en el tejido foliar o radical muerto fue considerada como un indicador directo de la proficiencia de reabsorción de dicho elemento (Killingbeck 1996).

Los cambios en el contenido de materia seca asociados a la traslocación durante la senescencia pueden enmascarar cambios netos en la concentración de nutrientes en los tejidos, lo cual crea un potencial problema con los valores de eficiencia y proficiencia de reabsorción de nutrientes calculados a partir de los cambios en sus concentraciones (van Heerwaarden et al. 2003). No obstante, las plantas con alto contenido de material estructural (i.e., fibra, lignina) son las que presentan los menores cambios en el contenido de materia seca durante el proceso de senescencia (Chapin 1980), y -en general- las gramíneas perennes del Caldenal presentan un contenido relativamente alto de material estructural en hojas y raíces (Moretto et al. 2001; Distel et al. 2005) en comparación con especies anuales o perennes de ambientes mésicos. Asimismo, las pérdidas de nutrientes por lixiviación y mineralización pueden restar certeza a la cuantificación de la eficiencia y proficiencia de reabsorción de nutrientes. Una forma de atenuar dicho efecto, y que fue tenido en cuenta en el presente estudio, es mediante el análisis de material muerto reciente, siendo esto más difícil de establecer en el caso de las raíces.

Dado que el interés primario fue la comparación entre especies los datos se analizaron mediante un análisis de la varianza (ANVA) doble, teniendo como fuentes de variación la especie, el tiempo y la interacción especie $x$ tiempo. Previo análisis, los datos porcentuales fueron transformados mediante la función arcoseno para satisfacer los requerimientos de normalidad y homocedasticidad del ANVA. Las medias se compararon mediante la prueba de Tukey $(P<0.05)$.

\section{Resultados}

La concentración de $\mathrm{N}$ en hojas vivas (Figura 1a) y hojas muertas (Figura 1b) varió con la especie $(P<0.001)$ y el tiempo $(P<0.001)$, y ambos factores interactuaron significativamente entre sí $(P<0.001)$. Durante algunos meses, la concentración de $\mathrm{N}$ en hojas vivas (junio y agosto) y en hojas muertas (agosto y septiembre) fue mayor en $P$. ligularis que en $N$. tenuissima, mientras que en el resto la diferencia no resultó significativa. La concentración de $\mathrm{N}$ en raíces vivas (Figura 1c) y raíces muertas (Figura $1 \mathrm{~d}$ ) también varió con la especie $(P<0.001)$ y el tiempo $(P<0.001)$, $\mathrm{y}$ ambos factores interactuaron de manera significativa $(P<0.001)$. No obstante, en todas las fechas de medición la concentración de $\mathrm{N}$ en ambos compartimientos (raíces vivas y raíces muertas) fue mayor en $P$. ligularis que en N. tenuissima.

La eficiencia de reabsorción de $\mathrm{N}$ a nivel foliar (Figura 2a) fue similar $(P>0.05)$ en ambas 
(a)
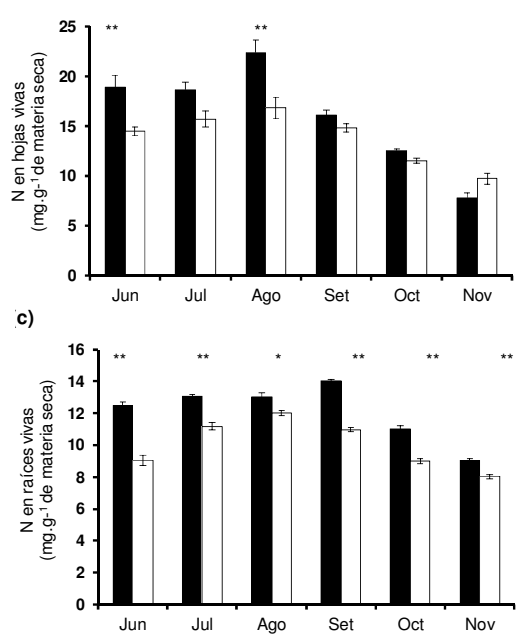

(b)

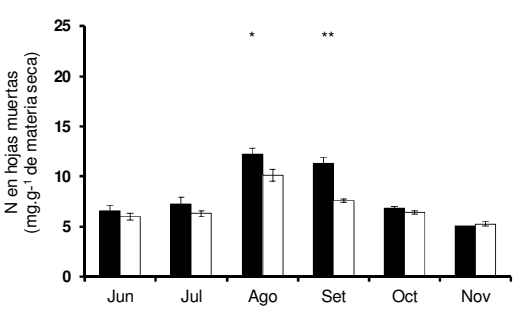

(d)

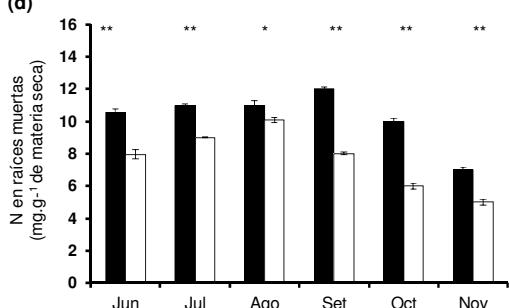

Figura 1. Variación temporal de la concentración nitrógeno $(\mathrm{N})$ en (a) hojas vivas, (b) hojas muertas, $(\mathrm{c})$ raíces vivas, y $(\mathrm{d})$ raíces muertas. Las barras verticales representan $\pm 1 \mathrm{EE}$ de la media $(n=10)$. Para cada fecha, los asteriscos indican diferencia significativa entre las especies, $P<0.05\left(^{*}\right) \mathrm{O}$ $P<0.01^{(* *)}$.

Figure 1. Temporal variation in the concentration of nitrogen (N) in (a) live leaves, (b) dead leaves, (c) live roots, and $(\mathrm{d})$ dead roots. Vertical bars represent $\pm 1 \mathrm{SE}$ of the mean $(n=10)$. For each date, asterisks indicate significant difference between species $P<0.05\left(^{*}\right)$ or $P<0.01\left(^{* *}\right)$. especies, aunque varió $(P<0.001)$ a través del tiempo y la interacción entre ambos factores fue significativa $(P<0.001)$. En septiembre, la reabsorción de $\mathrm{N}$ fue mayor en $N$. tenuissima, mientras que en las restantes fechas de medición no se registró diferencia entre ambas especies. La eficiencia de utilización de $\mathrm{N}$ a nivel foliar (Figura $2 \mathrm{~b}$ ) varió con la especie $(P<0.001)$ y el tiempo $(P<0.001)$, y ambos factores interactuaron $(P<0.05)$. En septiembre, la eficiencia de utilización de $\mathrm{N}$ a nivel foliar fue mayor en $N$. tenuissima, mientras que en las restantes fechas de medición la diferencia no fue significativa. La eficiencia de reabsorción de $\mathrm{N}$ a nivel radical (Figura 2c) varió con la especie $(P<0.001)$ y en el tiempo $(P<0.001)$, y ambos factores interactuaron significativamente $(P<0.001)$. En septiembre, octubre y noviembre, la reabsorción de $\mathrm{N}$ a nivel radical fue mayor en $N$. tenuissima, mientras que en las restantes fechas de medición no se registró diferencia entre ambas especies. La eficiencia de utilización de $\mathrm{N}$ a nivel radical (Figura $2 \mathrm{~d}$ ) varió con la especie $(P<0.001)$ y el tiempo $(P<0.001)$, y ambos factores interactuaron de manera significativa $(P<0.001)$. En agosto, la eficiencia de utilización de $\mathrm{N}$ fue similar en ambas especies, mientras que en las restantes fechas de medición la eficiencia de utilización de $\mathrm{N}$ a nivel radical fue mayor en $\mathrm{N}$. tenuissima que en $P$. ligularis.

\section{DisCUSIÓN}

Los resultados obtenidos fueron parcialmente consistentes con la hipótesis de una mayor eficiencia y/o proficiencia de reabsorción y de utilización $\mathrm{N}$ en $N$. tenuissima (baja palatabilidad) que en $P$.
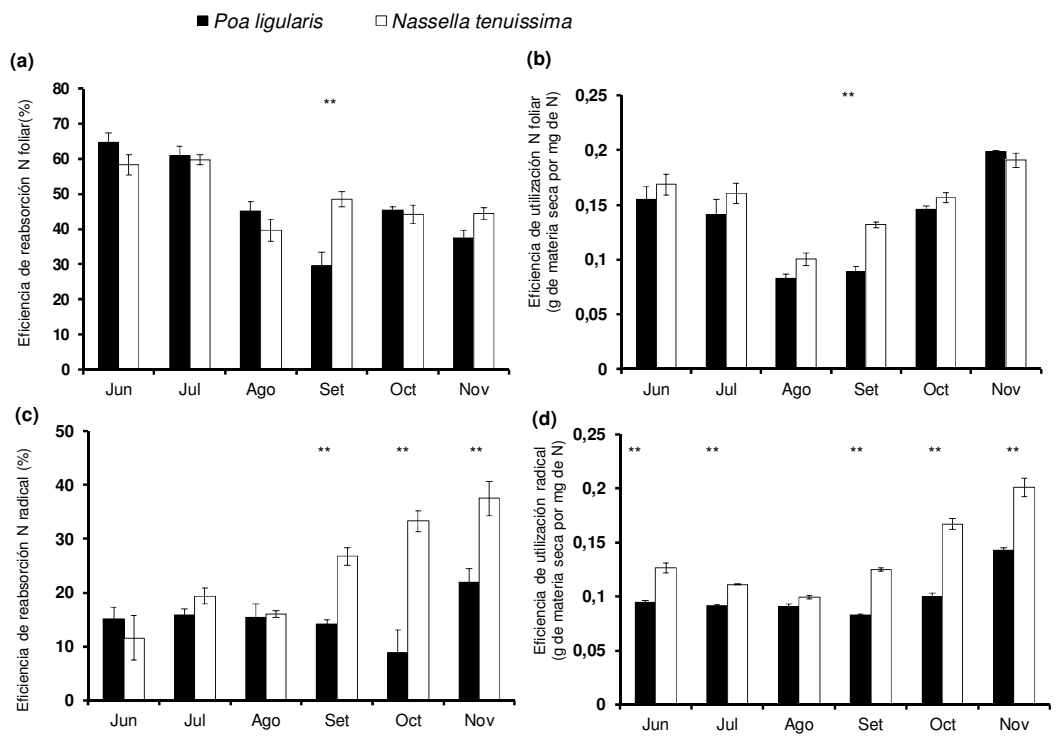

Figura 2. Variación temporal de la eficiencia reabsorción de nitrógeno $(\mathrm{N})$ (a) a nivel foliar y (b) a nivel radical, y de la eficiencia de utilización de $\mathrm{N}(\mathrm{c})$ a nivel foliar y $(\mathrm{d})$ a nivel radical. Las barras verticales representan $\pm 1 \mathrm{EE}$ de la media $(n=10)$. Para cada fecha, los asteriscos $\left.{ }^{* *}\right)$ indican diferencia significativa, entre especies $P<0.01$.

Figure 2. Temporal variation in nitrogen $(\mathrm{N})$ resorption efficiency at (a) leaf level and at (b) root level, and in $\mathrm{N}$ use efficiency at (c) leaf level and at (d) root level. Vertical bars represent \pm 1 $\mathrm{SE}$ of the mean $(\mathrm{n}=10)$. For each date, asterisks (**) indicate significant difference between species $P<0.01$. 
ligularis (alta palatabilidad), ya que para todas las variables analizadas se detectó una interacción significativa entre especie y tiempo (meses). No obstante, cuando existieron diferencias (en términos de eficiencia y/o proficiencia de reabsorción de $\mathrm{N}$ y eficiencia de utilización N), fueron favorables a la especie de baja palatabilidad. La conclusión señalada estaría limitada a las condiciones hídricas que se registraron durante el periodo de mediciones. Trabajos sobre el tema (Huang et al. 2009; Lü \& Han 2010) sugieren que el proceso de reabsorción de nutrientes es afectado por la interacción entre la identidad de las especies, la disponibilidad de nutrientes y la disponibilidad de agua. En años húmedos (como el del presente estudio) cabría esperar una reducción en la reabsorción de N (Lü y Han 2010).

La mayor proficiencia de reabsorción de $\mathrm{N}$ promedio observada en la especie de baja palatabilidad concuerda con resultados obtenidos en la comparación entre $P$. ligularis, Nassella tenuis (palatable) y Jarava speciosa (sinónimo Stipa speciosa, baja palatabilidad) en la Región del Monte Austral de nuestro país (Campanella \& Bertiller 2011). Sin embargo, en términos de eficiencia de reabsorción de $\mathrm{N}$ no hubo coincidencia ya que en el trabajo citado se observó una menor eficiencia de reabsorción de $\mathrm{N}$ en la especie de baja palatabilidad. De todas maneras, existe consenso en que la característica seleccionada para la conservación de nutrientes ha sido la concentración mínima del nutriente en el tejido senescente (proficiencia de reabsorción) más que la proporción del nutriente reabsorbido al momento de la senescencia (eficiencia de reabsorción) (Killingbeck 1996; Wright \& Westoby 2003). A menor concentración de un nutriente en el tejido senescente, mayor el tiempo de residencia del mismo en la planta y mayor la probabilidad de supervivencia en ambientes pobres en nutrientes.

Los valores promedio de proficiencia de reabsorción de $\mathrm{N}$ a nivel foliar en $P$. ligularis (8.22 $\mathrm{mg} / \mathrm{g})$ y $N$. tenuissima $(6.95 \mathrm{mg} / \mathrm{g})$ estuvieron cercanos a los valores ( $\leq 7.0 \mathrm{mg}$ ) g) que distinguen a las especies altamente proficientes en la reabsorción de N (Killingbeck 1996), particularmente en el caso de la especie de baja palatabilidad. También resultaron similares a valores promedio registrados para $S$. ichu $(7.0 \mathrm{mg} / \mathrm{g})$, característica de ambientes relativamente infértiles, y $S$. brachychaeta $(8.9 \mathrm{mg} / \mathrm{g})$, característica de ambientes relativamente fértiles, en el mismo sistema en estudio (Distel et al. 2003). Por el contrario, superaron valores promedio $(\leq 5.0$ $\mathrm{mg} / \mathrm{g})$ obtenidos para gramíneas perennes $(P$. ligularis, S. tenuis, S. speciosa) de la Región del Monte Austral (Carrera et al. 2000; Carrera et al. 2003; Bertiller et al. 2005), con condiciones edafoclimáticas menos favorables que las del sistema en estudio. En el caso de las especies del Monte Austral no se observaron diferencias en proficiencia de reabsorción de $\mathrm{N}$ entre las especies palatables (P. ligularis, $N$. tenuis) y la especie de baja palatabilidad (S. speciosa). Por otra parte, los valores medios de concentración de $\mathrm{N}$ en raíces muertas de $P$. ligularis (10.2 $\mathrm{mg} / \mathrm{g})$ y $N$. tenuissima $(7.6 \mathrm{mg} / \mathrm{g})$ estuvieron comprendidos en el rango de valores (5.1 a $11.9 \mathrm{mg} / \mathrm{g}$ ) correspondiente a varias especies de gramíneas nativas de América del Norte y de América del Sur (Vivanco \& Austin 2006), aunque en este trabajo no se discriminó entre raíces vivas y raíces muertas.

Los valores medios de eficiencia de reabsorción de $\mathrm{N}$ en hojas de $P$. ligularis (47\%) y N.tenuissima (49\%) estuvieron comprendidos en el rango de valores (35-60\%) para especies graminoides informado en un trabajo de revisión de Aerts \& Chapin (2000), pero fueron inferiores a valores promedios registrados en gramíneas perennes de la Región del Monte Austral (65-80\%) (Carrera et al. 2000; Carrera et al. 2003; Bertiller et al. 2005). Por otra parte, los valores medios de eficiencia de reabsorción de $\mathrm{N}$ en raíces de $P$. ligularis (15\%) y $N$. tenuissima (24\%) contrastaron con la escasa o nula reabsorción de $\mathrm{N}$ en raíces previamente cuantificada (Aerts et al. 1992) o comúnmente asumida (Chapin et al. 2002, pág. 192). La reabsorción de $\mathrm{N}$ a nivel radical observada, aunque de menor magnitud que la medida a nivel foliar, contribuiría en forma significativa a la economía de $\mathrm{N}$ de las especies, en razón de la abundante producción de biomasa radical de las gramíneas perennes en el sistema en estudio (puede llegar a septuplicar a la biomasa aérea) y la alta tasa de recambio de la misma (en el orden del 2/3 anual) (Distel \& Fernández 1986).

Los valores promedio de eficiencia de uso de $\mathrm{N}$ a nivel foliar y radical de $P$. ligularis (0.13 y $0.10 \mathrm{~g} \mathrm{MS} / \mathrm{mg} \mathrm{N}$, respectivamente) y $N$. tenuissima (0.15 y $0.14 \mathrm{~g} \mathrm{MS} / \mathrm{mg} \mathrm{N}$, respectivamente) estuvieron comprendidos por el rango de variación (0.08-0.17) de eficiencia de uso de $\mathrm{N}$ a nivel foliar para especies graminoides informado en el trabajo de revisión de Aerts \& Chapin (2000). La menor o similar concentración de nitrógeno en hojas y raíces vivas de $N$. tenuissima, conjuntamente con una eficiencia de reabsorción de $\mathrm{N}$ similar 
o mayor, resultó en una similar o mayor eficiencia de uso de $\mathrm{N}$ en $\mathrm{N}$. tenuissima en distintos momentos del ciclo de crecimiento. En este sentido, resulta necesario considerar que la ecuación de cálculo de la eficiencia de uso de $\mathrm{N}$ utilizada en este estudio (Vitousek 1982) no contempla el tiempo medio de residencia del nutriente en los tejidos vegetales (Berendse \& Aerts 1987), por lo cual los resultados de eficiencia de uso de $\mathrm{N}$ podrían ser conservadores, en particular en el caso de la especie de palatabilidad baja. Las gramíneas de baja palatabilidad tienen tejidos longevos (Cerqueira et al. 2004; Campanella \& Bertiller 2011), lo cual contribuye a aumentar el tiempo de residencia de los nutrientes en la planta y -por lo tanto- la economía de uso de los mismos. La mayor eficiencia de uso de $\mathrm{N}$ de $N$. tenuissima es consistente con la hipótesis de su adaptación a condiciones de infertilidad (Chapin et al. 2002).

La senescencia foliar (Distel \& Fernández 1986) y radical (Distel \& Fernández 1988) es un proceso gradual en las especies en estudio. Nuestros resultados mostraron variaciones temporales significativas para todas las variables analizadas. Estas variaciones estarían determinadas por el grado de disponibilidad de destinos con demanda elevada de nutrientes (e.g., tejidos en crecimiento, órganos de almacenaje) y por factores ambientales que influencian el crecimiento de las plantas (Pugnaire \& Chapin 1993; Lü et al. 2012). Sumado a esto, hubo una interacción significativa entre tiempo y especie, tanto en la proficiencia como en la eficiencia de reabsorción de $\mathrm{N}$ en hojas y raíces, lo cual podría estar relacionado con diferencias en la maduración de ambas especies ( $P$. ligularis inicia la floración en septiembre, mientras que $N$. tenuissima la inicia en octubre). Este tipo de variación temporal es común en los estudios sobre el tema, constituyendo un serio problema en comparaciones interespecíficas puntuales (Killingbeck 1996). En este sentido el presente trabajo cuenta con la ventaja de haber incluido numerosas mediciones a lo largo del ciclo de crecimiento de las especies, a diferencia de otros que sólo presentan mediciones puntuales.

Las diferencias entre las especies en estudio en cuanto a la eficiencia y/o la proficiencia de reabsorción de $\mathrm{N}$ podrían tener consecuencias sobre las tasas de descomposición y mineralización del N. La existencia de una retroalimentación positiva entre condiciones de infertilidad y adaptación de las plantas a tales condiciones ha sido resaltada por varios autores (Jones et al. 1994; Aerts 1995; Wedin 1995). No obstante, estudios previos conducidos en el mismo sitio de estudio no mostraron una diferenciación clara en las tasas de descomposición y de mineralización del $\mathrm{N}$ entre gramíneas perennes de diferente palatabilidad (Moretto et al. 2001; Andreoli \& Distel 2008). Las gramíneas perennes $C_{3}$ del Caldenal presentan una variación limitada en la composición química de hojas y raíces senescentes (Andrioli \& Distel 2008), en comparación con las variaciones existentes entre gramíneas $C_{3}$ y gramíneas $C_{4}$ (Wedin \& Tilman 1990; Gijsman et al. 1997; Urquiaga et al. 1998). Además, el funcionamiento de los pastizales del Caldenal está frecuentemente limitado por escasez de agua (Distel \& Fernández 1987) y, en dichas condiciones, la influencia de la especie sobre la dinámica del $\mathrm{N}$ en el suelo estaría reducida (Burke et al. 1997; Burke et al. 1998; Hooper \& Johnson 1999). Ambos factores en conjunto contribuirían a explicar la similitud en la tasa de descomposición y de mineralización del $\mathrm{N}$ de las gramíneas perennes de diferente palatabilidad, a pesar de las diferencias en la eficiencia y/o proficiencia de reabsorción de $\mathrm{N}$ que pudiera existir entre ellas, como las observadas en el presente estudio.

Agradecimientos: A S. Amán por su invaluable ayuda en los muestreos de campo.

\section{BIBLIOGRAFÍA}

Aerts, R; B Wallen \& N Malm. 1992. Growth-limiting nutrients in Sphagnum-dominated bogs subjected to low and high atmospheric nitrogen supply. Journal of Ecology, 80:131-140.

AERTs, R. 1995. The advantages of being evergreen. Trends in Ecology and Evolution, 10:402-407.

AERTS, R. 1996. Nutrient resorption from senescing leaves of perennials: are there general patterns? Journal of Ecology, 84:597-608.

AERTS, R \& F Chapin III. 2000. The mineral of wild plants revisited: A re-evaluation of processes and patterns. Advances in Ecological Research, 30:2-67.

AndRioli, R \& RA Distel. 2008. Litter quality of $\mathrm{C}_{3}$ perennial grasses and soil inorganic nitrogen in a semiarid rangeland of central Argentina. Journal of Arid Environments, 72:1684-1689.

BerEndSE \& R Aerts. 1987. Nitrogen-use-efficiency: A biologicallymMeaningful definition?. Functional Ecology, 1(3): 293-296.

Bertiller, MB; CL Sain; AL Carrera \& DN Vargas. 2005. Patterns of nitrogen and phosphorous conservation in dominant perennial grasses and shrubs across an aridity gradient in Patagonia. Journal of Arid Environment, 62:209-223.

Bонм, W. 1979. Methods of studying root systems. SpringerVerlag, New York.

Burke, I; W Lauenroth \& WJ Parton. 1997. Regional and temporal variation in net primary production and nitrogen mineralization in grasslands. Ecology, 78:1330-1340.

Burke, IC; WK Lauenroth; J Steenson; M Gutmann; 
WJ PARTON; ET AL. 1998. Environmental controls over land use in the Central grasslands region of the United States. Canberra (Australia): Global Change and Terrestrial Ecology Abstracts Core Project Office.

Cabrera, AL. 1976. Regiones Fitogeográficas Argentinas. En: Enciclopedia Argentina de Agricultura y Jardinería. Tomo II. Fascículo 1. ACME, Buenos Aires.

Caldwell, MM. 1979. Root structure: the considerable cost of below ground function. Pp. 408-432 en: Solbrig, OT; S Jain; GB Johnson \& PH Raven (eds.). Topics in Plant Population Biology. Columbia University Press, New York.

Campanella, MV \& MB Bertiller. 2011. Is N-resorption efficiency related to secondary compounds and leaf longevity in coexisting plant species of the arid Patagonian Monte, Argentina? Austral Ecology, 36:395-402.

CANO, E. 1988. Pastizales naturales de La Pampa. Descripción de las especies más importantes. Tomo 1. Convenio AACREA-Provincia de La Pampa.

Carrera, AL; CL Sain \& MB Bertiller. 2000. Patterns of nitrogen conservation in shrubs and grasses in the Patagonian Monte, Argentina. Plant and Soil, 224:185-193.

Carrera, AL; MB Bertiller; CL Sain \& MJ Mazzarino. 2003. Relationship between plant nitrogen conservation strategies and the dynamics of soil nitrogen in the arid Patagonian Monte, Argentina. Plant Soil, 255:595-604.

Cerqueira, E; AM SÁEnz \& CM Rabotnikof. 2004. Seasonal nutritive value of native grasses of Argentine Calden Forest Range. Journal of Arid Environments, 54:645-656.

Chapin III, F. 1980. The mineral nutrition of wild plants. Annual Review of Ecology and Systematics, 11:233-260.

Chapin III, F; PA Matson; A Harold \& E Money. 2002. Principles of terrestrial ecosystem ecology. Springer, NewYork.

DisTel, RA. 1987. Crecimiento aéreo y radical, germinación y supervivencia en Piptochaetium napostaense (Speg.) Hack y Stipa tenuis Phil. Tesis de Maestría, Universidad Nacional del sur, Bahía Blanca (Argentina).

Distel, RA \& DV Peláez. 1985. Fenología de algunas especies del Distrito del Caldén (Prosopis caldenia Burk.). IDIA Sept.-Dic.:35-40.

Distel, RA \& OA FERnÁndez. 1986. Productivity of Stipa tenuis Phil. and Piptochaetium napostaense (Speg) Hach. in semi-arid Argentina. Journal of Arid Environment, 11:93-6.

DisTEL, RA \& OA FERNÁNDEZ. 1987. Leaf water potential trends in three grasses native to semiarid Argentina. Journal of Range Management, 40:203-207.

Distel, RA \& O A Fernández. 1988. Dynamics of root growth and decay in two grasses native to semi-arid Argentina. Australian Journal of Ecology, 13:327-336.

Distel, RA \& RM Bóo. 1996. Vegetation states and transitions in temperate semi-arid rangelands of Argentina. Pp. 117-118 en: West, N (ed.). Rangelands in a Sustainable Biosphere. Denver: Society for Range Management. USA.

Distel, RA; AS Moretto \& NG Didoné. 2003. Nutrient resorption from senescing leaves in two Stipa species native to central Argentina. Austral Ecology, 28:210-215.

Distel, RA; NG Didoné \& AS MoretTo. 2005. Variations in chemical composition associated with tissue ageing in palatable and unpalatable grasses native to central Argentina. Journal of Arid Environment, 62:351-357.

Gijsman, AJ; HF Alarcón \& RJ Thomas. 1997. Root decomposition in tropical grasses and legumes, as affected by soil texture and season. Soil Biology and Biochemistry, 29:1443-1450.

GrIME, JP. 1979. Plant strategies and vegetation processes. Wiley \& Sons, Chichester.

GRIME, JP. 2001. Plant strategies, vegetation processes, and ecosystem properties. Wiley, New York.

HOOPER, DU \& L JOHNSON. 1999. Nitrogen limitation in dryland ecosystems: responses to geographical and temporal variation in precipitation. Biogeochemistry, 46:247-293.

HuAnG, JY, HL Yu, LH Li, ZY YuAN \& S Bartels. 2009. Water supply changes $\mathrm{N}$ and $\mathrm{P}$ conservation in a perennial grass Leymus chinensis. Journal of Integrative Plant Biology, 51:1050-1056.

INTA; Provincia de La PAMPa \& Universidad Nacional de LA PAMPA. 1980. Inventario integrado de los recursos naturales de la Provincia de La Pampa. INTA, Buenos Aires.

InstITUTODEBOTÁNICADARWINION. 2009. Flora del Conosur. Catálogo de Plantas Vasculares. http:/ / www.darwin.edu.ar.

Jones, CG; JH LAWTON \& M SHACHAK. 1994. Organisms as ecosystem engineers. Oikos, 69:373-86.

KillingBecK, K. 1996. The terminological jungle revisited: making a case for use of the term resorption. Oikos, 46:263-264.

LÜ, XT \& XG HAN. 2010. Nutrient resorption responses to water and nitrogen amendment in semi-arid grassland of Inner Mongolia, China. Plant and Soil, 397:481-491.

LÜ, XT; GT FREschet; DFB FlynN \& GH XING. 2012. Plasticity in leaf and stem nutrient resorption proficiency potentially reinforces plant-soil feedbacks and microscale heterogeneity in a semi-arid grassland. Journal of Ecology, 100:144-150.

MoORE, RP. 1962. Tetrazolium as a universally accepted quality test for viable seed. Proceedings of the International Seed Testing Association, 27:795-805.

Moretto, AS; RA Distel \& NG Didoné. 2001. Decomposition and nutrient dynamic of leaf litter and roots from palatable and unpalatable grasses in a semiarid grassland. Applied Soil Ecology, 18:31-37.

Oestertag, R \& SE Hobbie. 1999. Early stages of root and leaf decomposition in Hawaiian forest: effects of nutrient availability. Oecologia, 121:564-573.

Pugnaire, FI \& FS Chapin. 1993. Controls over nutrient resorption from leaves evergreen Mediterranean species. Ecology, 74:124-129.

Urquiaga, S; G Cadish; BJ Alves; RM Boddey \& KE GILLER. 1998. Influence of decomposition of tropical forage species on the availability of soil nitrogen. Soil Biolology and Biochemistry, 30:2099-2106.

van Heerwaarden LM; S Toet \& R Aerts. 2003. Current measures of nutrient resorption efficiency lead to a substantial underestimation of real resorption efficiency: facts and solutions. Oikos, 101:664-669.

Vitousek, P. 1982. Nutrient cycling and nutrient use efficiency. American Naturalist, 119:553-572.

VIVANCO, L \& AT AUsTIN. 2006. Intrinsic species effects on leaf litter and root decomposition: a comparison of temperate grasses from North and South America. Oecologia, 150:97-107.

Wedin, A \& D Tilman. 1990. Species effects on nitrogen cycling: a test with perennial grasses. Oecologia, 84:433-441.

Wedin, DA. 1995. Species, nitrogen, and grassland dynamics: the constraint of stuff. Pp. 253-262 en: Jones, CG \& LH Lawton (eds.). Linking Species and Ecosystems. Chapman \& Hall, New York.

Wedin, DA. 1999. Nitrogen availability, plant-soil feedbacks and grassland stability. Pp. 193-197 en: Eldridge, D\&D Freudenberger (eds.). People and Rangelands Building the Future. Proceedings of the VI International Rangeland Congress, Volume 1, Townsville.

Wright IJ \& M Westoby. 2003. Nutrient concentration, resorption and lifespan: leaf traits of Australian sclerophyll species. Functional Ecology, 17:10-19. 